N. E. Piskunov, W. W. Weiss, D. F. Gray, eds.

\title{
NLTE Radiative Transfer in the Extended Atmospheres and Winds of Cool Stars
}

\author{
Philip D. Bennett ${ }^{1}$, Graham M. Harper, and Alexander Brown \\ Center for Astrophysics and Space Astronomy, University of Colorado, \\ Boulder, CO 80309-0389 \\ Jeffrey L. Linsky \\ JILA, University of Colorado and NIST, Boulder, CO 80309-0440
}

\begin{abstract}
.
The mechanism responsible for driving the ubiquitous winds of cool giant and supergiant stars remains to be established. To this end, we are constructing semi-empirical models of the extended outer atmospheres ('chromospheres') and winds of selected red supergiants. These models are constrained by analyses of the UV line spectra of single stars, and of red supergiants in binaries that eclipse their main-sequence companions: the $\zeta$ Aur and VV Cep stars. These detached binaries are well-separated, with no evidence of mass transfer. The C II] $2325 \AA$ line profiles of the binaries are similar to those of comparable single stars, suggesting that the chromospheres remain relatively unperturbed by binarity. However, it is unclear how much binarity disturbs the wind: binary observations suggest a gradual acceleration $(\beta \sim 3)$, but line profile analyses of single red supergiants imply a rapid acceleration $(\beta<1)$. To date, we have obtained extensive series of HST/GHRS and STIS observations of three eclipsing red supergiant binaries: $\zeta$ Aur, HR 2554 and VV Cep. In this paper, we focus on $\zeta$ Aur, and present observations and modelling results for this eclipsing binary.
\end{abstract}

\section{Introduction}

We are constructing semi-empirical models of the outer atmospheres of late-type (red) supergiant (F-M I or II) stars with the goal of understanding the wind acceleration and resulting mass loss process in these stars - a question which has important implications for the evolution of intermediate mass and massive stars, and the chemical evolution of the galaxy. Our main objective is to clearly establish the physical conditions present in chromospheres and winds of red supergiants (RSGs) using the superb spectroscopic data now available from the Hubble Space Telescope (HST) GHRS and STIS spectrographs, and the FUSE

\footnotetext{
${ }^{1}$ Adjunct Professor, Dept. of Astronomy \& Physics, Saint Mary's University, Halifax, N.S. B3H 3C3, Canada
} 
satellite, and also from ground-based observations in the optical (DAO) and the radio continuum (VLA). To this end, we are pursuing two complementary approaches:

- Detailed analysis and synthesis of the ultraviolet spectrum of single RSGs, which provides constraints on physical conditions in the upper photosphere and chromosphere

- Analysis of the ultraviolet spectra of RSG binaries with main-sequence companions, which constrains the chromosphere and wind regimes lying outside the line-forming region in single stars.

In this paper, we focus on the latter approach: using the early-type companions of red supergiants in eclipsing binary systems to probe winds of the supergiant primaries. These binaries (the $\zeta$ Aur or VV Cep stars) offer substantial advantages in probing stellar winds:

- there are many more photons available in the UV where the most useful line diagnostics are found,

- information can be obtained along localized lines of sight that permit spatial structure to be resolved,

- the source of the UV photons (the hotter MS companion) is distinct from the source of the wind (the red supergiant), resulting in stronger and much less ambiguous wind features in the spectrum, and

- observations at different orbital phase angles provide direct information about possible 2-D structure and/or variability in time.

There are also disadvantages. The main ones are:

- the sample of suitable, bright, eclipsing binaries containing red supergiants is very limited: $\sim 10$ stars in the Bright Star Catalogue,

- the interaction between the supergiant's wind and the hot companion may substantially affect the wind acceleration process, and

- modelling the line formation in the wind for binaries is more complex because the radiative transfer problem is (at best) 2-D axisymmetric.

All of the binaries considered are detached systems in apparently primordial (usually eccentric) orbits without indication of prior mass transfer. The supergiant's wind far from the star is probably substantially modified from the winds of comparable single stars. These binary winds are heated and partially photoionized by the companion's bright UV continuum, and dynamically disturbed by the companion's gravitational field and orbital motion. However, the intrinsic UV chromospheric spectrum of the supergiants in binaries (as observed during eclipse) appears to be similar to the spectrum of comparable single supergiant stars. This suggests that the supergiant's atmosphere at the base of the wind remains relatively unperturbed by the presence of the companion star. The question of whether the wind acceleration process is also similar in the binaries and the single supergiants remains open. 
In this paper, we first provide a brief review of the $\zeta$ Aur binaries, and summarize our sequences of spectroscopic observations of these stars with the HST. We present HST observations of $\zeta \mathrm{Aur}^{2}$ showing the behavior of the spectrum at differing orbital phases. We discuss UV line formation in binary winds, for which the source of photons (the hot companion) is distinct from the source of the wind (the red supergiant primary). This property of the binary winds means that, even in the simplest models assuming spherically symmetric winds, the line radiative transfer solution requires solving a 2-D axisymmetric problem in a moving medium. Finally, we describe approximate numerical approaches to solving the line formation problem in these binaries.

\section{The $\zeta$ Aurigae Stars}

The $\zeta$ Aurigae stars are detached binaries with $\mathrm{G}$ or $\mathrm{K}$ supergiant stars that eclipse their early-type main sequence companions. The VV Cephei stars are similar systems with M supergiant primaries instead. These binaries have orbital periods of a few years and reasonably eccentric $(e \sim 0.2-0.4)$ orbits, reflecting a lack of tidal interaction or prior mass transfer. During eclipse, the observed C II] $2325 \AA$ and Al II] $2669 \AA$ lines are formed by collisional excitation in the chromospheres of the supergiant primaries, unlike nearly all other lines which scatter continuum radiation from the hot companion. The similarity of the line profiles and surface fluxes of these collisionally-excited chromospheric lines in $\zeta$ Aur $(\mathrm{K} 4 \mathrm{IB}+\mathrm{B} 5 \mathrm{~V})$ and in the comparable single supergiant, $\lambda$ Vel $(\mathrm{K} 4 \mathrm{Ib})$, strongly suggests that the chromospheres are not substantially affected by binarity, as noted by Eaton (1992).

Near eclipse, the line of sight to the hot secondary sweeps through the extended outer atmosphere of the primary. A "chromospheric" absorption spectrum from material in the supergiant primary's outer atmosphere is seen superimposed upon the continuum of the hot star. By repeated observation, the spatial structure of the supergiant's chromosphere can be mapped in a very direct manner. The orbit of $\zeta$ Aur is shown to scale in Figure 1.

The usefulness of the $\zeta$ Aurigae binaries for studying the chromospheres and winds of late-type stars has long been recognized; a classic curve of growth analysis of the pure absorption spectrum seen near eclipse was done nearly a halfcentury ago by Wilson \& Abt (1954). A review of the extensive early optical studies is given by Wright (1970). Observation in the ultraviolet at $\lambda<3000 \AA$ is especially advantageous because there the flux contribution from the cool primary is small or negligible. Furthermore, the main-sequence companions are much smaller in size than their evolved supergiant primaries. These properties make the $\zeta$ Aurigae secondaries nearly ideal "light probes" of the outer atmospheres of the primary stars. The launch of $I U E$ in 1978 rendered the UV spectra of these stars readily accessible to analysis for the first time. Consequently, a large number of observations of the $\zeta$ Aurigae stars have been obtained over the 18 year lifetime of $I U E$. The data obtained have been used to construct

\footnotetext{
${ }^{2}$ Based on observations with the NASA/ESA Hubble Space Telescope obtained at the Space Telescope Science Institute, which is operated by the Association of Universities for Research in Astronomy, Incorporated, under NASA contract NAS5-26555
} 
GHRS Observation Epochs •

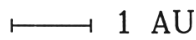

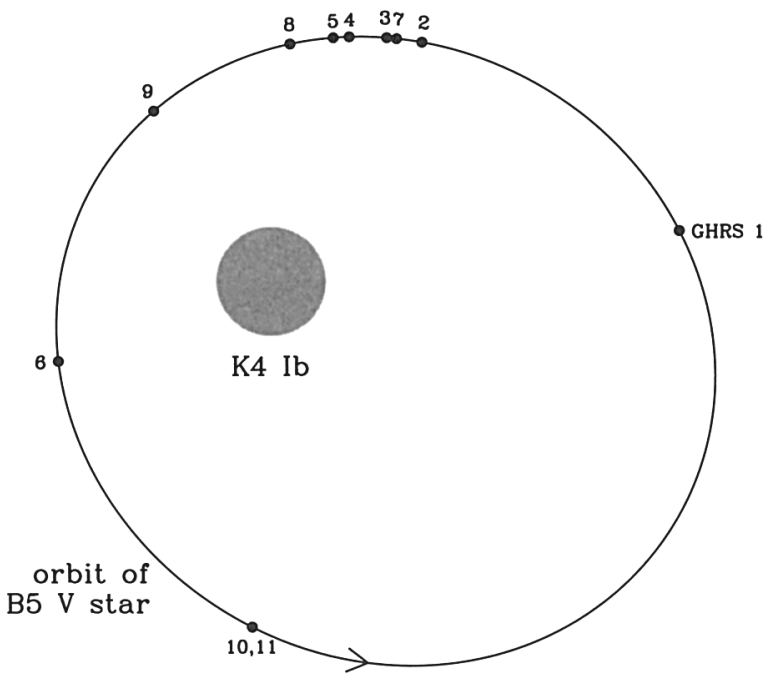

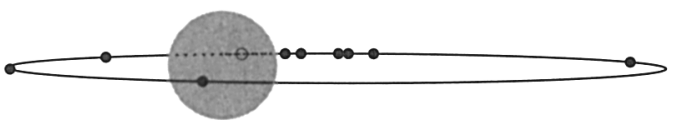

Figure 1. The relative orbit of $\zeta$ Aur, as viewed in its plane (top), and as projected on the sky (bottom), with the orbital phases of our HST/GHRS observations indicated. The figure is to scale, except that the size of the main-sequence secondary star has been enlarged for clarity. The line of sight to the earth (top image) is vertically down the page.

models of the chromosphere and wind of these stars, e.g. for $\zeta$ Aur alone, spectroscopic analyses based on $I U E$ observations include those of Chapman (1981), Stencel \& Chapman (1981), Ahmad et al. (1983), Che et al. (1983), Schröder (1985,1986), Ahmad (1986), Ahmad \& Stencel (1988), Kuin \& Ahmad (1989), Eaton (1992,1993), and Kirsch \& Baade (1994). Hempe (1982) developed an Sobolev (SEI) approach to solve the radiative transfer problem in the binary's circumstellar envelope, and Baade (1990) developed an exact solution of the 2-D axisymmetric line transfer problem using an integral operator method. However, all of these analyses suffered from the low S/N (typically $\sim 15$ ), low spectral resolution $\left(25 \mathrm{~km} \mathrm{~s}^{-1}\right)$ and low dynamic range of the IUE spectra, which are inadequate for quantitative line profile work. Recent optical observations of $\zeta$ Aur reported by Griffin et al. (1990) and analysis by Schröder et al. (1990) have better spectral resolution and somewhat better $\mathrm{S} / \mathrm{N}$ than the IUE data. 
Nevertheless, the lack of strong diagnostics of the wind region in these optical spectra limit their usefulness to studies of the denser chromosphere.

In contrast, the Goddard High Resolution Spectrograph (GHRS), and its replacement instrument on-board HST, the Space Telescope Imaging Spectrograph (STIS), have high spectral resolution, high S/N, and, for STIS, large spectral coverage, making UV binary eclipse spectroscopy a direct and extremely powerful technique in the study of stellar chromospheres and winds. A recent analysis of $\zeta$ Aur, based on part of the HST/GHRS data set, was done by Baade et al. (1996).

\section{Observations with the Hubble Space Telescope}

To date, we have obtained and analyzed observations of several binary systems containing red supergiants, along with observations of comparable single supergiants. A complete list of HST/GHRS and STIS observations of red supergiants in binaries, is shown in Table 1. Of these binaries, we have observed the total eclipse spectrum of $\zeta$ Aur, VV Cep, and $32 \mathrm{Cyg}$, central eclipse of HR 2554 (the eclipse is partial), and the eclipse of $31 \mathrm{Cyg}$ will, hopefully, be observed next year with HST/STIS.

In terms of modelling stellar winds, our focus so far has been on the wellobserved binary system $\zeta$ Aur and its single star spectral proxy $\lambda$ Vel. However, our longterm spectroscopic program to monitor the eclipse and egress of the long-period (20.3 yrs) eclipsing binary VV Cep is now essentially complete, and construction of detailed models of the chromosphere and wind of this supergiant, along with its spectral proxy $\alpha$ Ori, are in progress. In this paper, we focus on $\zeta$ Aur, and present observations, preliminary results and summarize the EPEI method under development for solving the 2-D axisymmetric radiative transfer problem in $\zeta$ Aur. The other binaries will be modelled in a similar manner.

\section{Ultraviolet Line Formation in the Binary Spectrum}

The formation of the ultraviolet spectrum in these binaries, even without significant contribution from the RSG primaries, is complex. The spectrum out of eclipse is dominated by the early-type continuum of the hot companion. This spectrum is a rather featureless flat continuum throughout much of the UV longward of $1400 \AA$, but shortward of this wavelength it becomes increasingly structured due to the presence of strong resonance lines of the abundant singly (and some doubly) ionized species, as well as the very broad Lyman- $\alpha$ line. The companions are generally rapid rotators, and are seen at high inclinations (near $90^{\circ}$ ) in the eclipsing systems, so the spectra show the full rotational broadening.

Near eclipse, the chromospheric absorption spectrum becomes evident, as shown in Figure 2. A forest of absorption lines, due to absorption in the RSG chromosphere along the line of sight to the hot companion, appear near eclipse and strengthen as eclipse approaches. Analysis of the growth of these absorption lines allows the density, temperature, velocity and ionization structure of the chromosphere to be mapped in great detail.

During eclipse, a strong emission line spectrum is seen, with a weak continuum present at shorter wavelengths $(<1300 \AA)$. Since the direct line of sight 


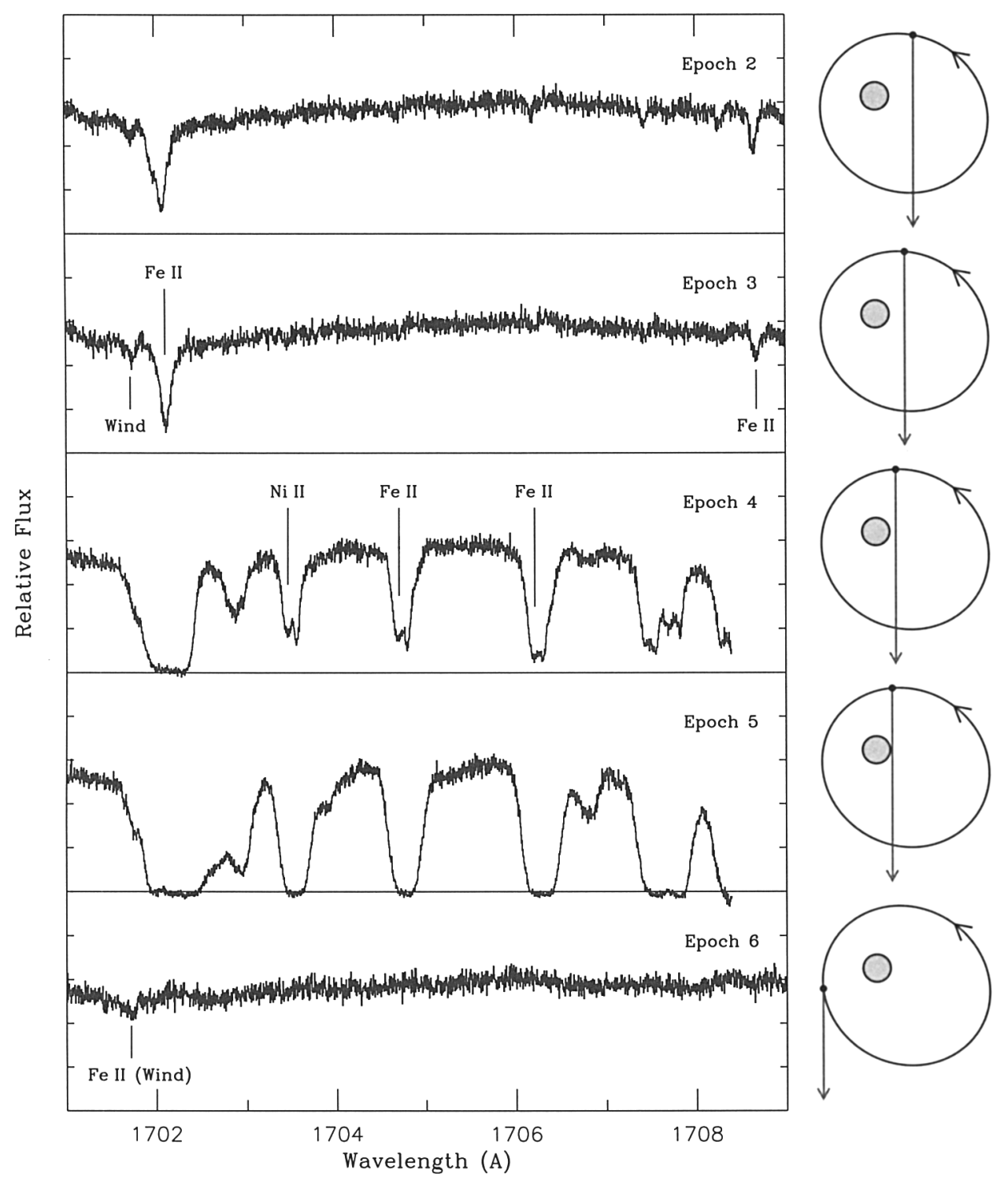

Figure 2. GHRS spectrum of $\zeta$ Aur near $1700 \AA$ showing chromospheric absorption near eclipse. Icons show the orbital configuration. 
Table 1. HST Ultraviolet Spectroscopy of Binary Red Supergiants

\begin{tabular}{rcclrl}
\hline Program & PI & Targets & Spectrum & Visits & Spectrograph \\
\hline & & & & & \\
3626 & Brown & $\zeta$ Aur & K4 Ib + B5 V & 5 & GHRS \\
5069 & Brown & $\zeta$ Aur & K4 Ib + B5 V & 1 & GHRS \\
5398 & Brown & HR 2554 & G6 II + A1 V & 8 & GHRS \\
5952 & Reimers & $\alpha$ Sco & M1 Iab + B1 V & 1 & GHRS \\
6069 & Brown & $\zeta$ Aur & K4 Ib + B5 V & 3 & GHRS \\
6515 & Brown & $\zeta$ Aur & K4 Ib + B5 V & 2 & GHRS \\
6288 & Brown & HR 2554 & G6 II + A1 V & 4 & GHRS \\
6289 & Lambert & $\epsilon$ Aur & F0 Ia + ? & 1 & GHRS(low-res) \\
6522 & Baade & 32 Cyg & K4 Ib + B5 V & 6 & GHRS \\
7269 & Bennett & VV Cep & M2 Iab + B0.5 V & 14 & STIS \\
8257 & Bennett & VV Cep & M2 Iab + B0.5 V & 2 & STIS \\
8257 & Bennett & KQ Pup & M2 Iab + B2 V & 1 & STIS \\
8257 & Bennett & HR 8164 & M1 Ib + B2 V & 1 & STIS \\
8779 & Bennett & VV Cep & M2 Iab + B0.5 V & 2 & STIS \\
9109 & Bennett & 31 Cyg & K4 Ib + B3 V & 2 & STIS \\
9231 & Bennett & VV Cep & M2 Iab + B0.5 V & 2 & STIS
\end{tabular}

to the hot companion is blocked, this spectrum is formed by (hot star) photons scattering in the chromosphere and wind of the RSG primary where the opacity is sufficiently high. Mostly, scattering occurs in the cores of lines arising from ground and metastable levels of the dominant ionization states. However, in the spectral region immediately longward of Lyman- $\alpha$, the Rayleigh scattering cross section of neutral hydrogen (here dominated by the red wing of Lyman- $\alpha$ ) is large, and gives rise to the scattered light continuum evident during eclipse. Of course, the scattered light contribution is always present, but is often overwhelmed by the direct contribution from the hot star's continuum out of eclipse. The scattered light spectrum is only obvious out of eclipse in the cores of strong lines, where the line profiles acquire a $\mathrm{P}$ Cygni character due to the combination of line-of-sight absorption superimposed on the scattered light contribution. A complete, self-consistent analysis of the wind requires modelling the radiative transfer and line formation problem, which is complex due to the lack of spherical symmetry.

Another contribution to the profile appears in a few of the hotter diagnostic lines, e.g., the C IV resonance doublet near $1550 \AA$. These lines show broad inverse P-Cygni profiles characteristic of high-velocity $\left(\sim 250 \mathrm{~km} \mathrm{~s}^{-1}\right)$ accretion, and display substantial profile variations on timescale of a few hours, for $\zeta$ Aur. The only plausible site for line formation involving these velocities and timescales is in an accretion region close to the early-type companion star. While the wind accretion contribution to the spectrum of $\zeta$ Aur is limited and is seen in only a very few lines, in other binaries with larger mass loss rates (e.g., VV Cep), the accretion contribution to the spectrum is substantial.

Finally, interstellar absorption is usually significant for neutral and singlyionized zero volt lines, and typically obliterates a portion of the stellar profile near line center. 


\section{Solving the Binary Radiative Transfer Problem}

Generally, the temperatures and densities in the circumstellar environments of these binaries are such that the non-metastable levels depopulate. Metastable level populations remain approximately Boltzmann for the denser chromosphere (e.g. Fe II lines), but begin to depopulate (e.g. Fe III lines) in the lower-density wind. Photons in the cores of lines with populated lower levels can be scattered by the appropriate absorbers. Then, the formation of the UV spectrum of the binaries is complex, with substantial contributions to the emergent flux from both direct (i.e. line of sight) and indirect (scattered) photon paths. Assuming the wind is spherical, the binary radiative transfer is a 2-D NLTE axially symmetric (about the binary axis) problem in a moving medium. For supergiants with low mass loss rates, the scattering contribution to the observed wind line profile is generally small for weak lines. However, for strong lines, and for all lines formed in winds of supergiants with high mass loss rates (e.g., VV Cep), the scattering component is substantial and often dominant.

The method of using the early-type companion to probe the structure of the supergiants's atmosphere near eclipse is very useful, and provides the only direct way of resolving structure, on small spatial scales, in a stellar atmosphere. Although very powerful, the "chromospheric eclipse" method is complicated by two factors. First, the supergiant has an observable, albeit weak, spectrum longward of $\sim 2300 \AA$, where the late-type continuum becomes obvious. Eclipse observations, or observations of comparable single stars, can be used to largely remove the contaminating primary spectrum. Second, it is necessary to consider the indirect component of line formation contributed by photons scattered by the supergiant's wind. There are several ways to address this problem:

- analyze weak lines near eclipse (i.e., at large absorption columns) and ignore the (small) scattering component.

- subtract the eclipse spectrum (which consists entirely of the wind-scattered component) from the nearby out-of-eclipse spectrum, since the geometry of the line formation problem is only slightly changed from the total eclipse configuration.

- solve the complete line formation problem and model the combined absorption and scattering components of the line profile.

- obtain a simpler, approximate solution of the line formation problem

Historically, the first approach, for which the analysis is simple, has been the method of choice. Our analyses, especially for VV Cep for which the mass loss is high, and the scattering component strong, have used the eclipse subtraction approach also. This has also been done by some optical observers (cf. Griffin et al. 1990) as a byproduct of separating the composite spectra. Incomplete subtraction of the wind-scattered component of the line profile becomes increasingly problematic with increasing orbital phase difference from eclipse, due to radial velocity and intensity mismatches between the eclipse and out-of-eclipse scattered light component. Also, in many cases, especially for HST/GHRS echelle observations (for which the spectral coverage is only $\sim 10 \AA$ per exposure), eclipse 
observations are not available to match lines observed out-of-eclipse. Therefore, complete analyses of the spectra of these binaries requires solving the full 2-D axisymmetric radiative transfer problem in the wind to compute both absorption and scattering components of the wind line profiles. This approach was pioneered by the Hamburg group: Che et al (1983) developed an SEI code to obtain approximate solutions of the line transfer problem for the binary winds. Baade (1990) developed an exact integral operator approach to handle the line formation problem in a general geometry, which was then specialized to the particular case of these binary stars. Baade et al. (1996) successfully applied this method to HST/GHRS observations of $\zeta$ Aur. Recently, there has been a resurgence of work in the field of $2-\mathrm{D}$ radiative transfer, with Busche \& Hillier (2000) and Dullemond \& Turolla (2000) independently developing solutions for the 2-D axisymmetric problem in spherical coordinates using short characteristic methods.

It is clear that we need to solve the full transfer problem if the maximum amount of information is to be extracted from the observed P-Cygni line profiles in these binaries. However, obtaining an exact solution of this $2-\mathrm{D}$ radiative transfer problem remains a difficult, computationally intensive task. This is especially so when the goal is to optimize the wind model parameter space for the solution that best reproduces observed wind profiles. This process requires solving the 2-D transfer problem repeatedly for different wind models, a very time-consuming approach when the exact solution is used. Furthermore, it should be realized that this "exact solution" is achieved for an approximate problem since the wind is almost certainly not spherical because of orbital motion. Given that the problem being solved is already an idealized description of reality, we believe it is entirely appropriate to forego an exact solution of the $2-\mathrm{D}$ radiative transfer problem in favor of a fast, robust, approximate solution.

We make the following assumptions in order to simplify the wind model:

- the wind, density, and other thermodynamic quantities are steady in time and spherically symmetric about the instantaneous current position of the supergiant star,

- the effect of orbital dynamics on the wind are ignored,

- all (UV) photons originate from the hot companion,

- 2-level atom or equivalent 2-level atom (ETLA),

- complete frequency redistribution (CRD),

- ground or metastable levels have Boltzmann populations while all other levels are depopulated,

With these assumptions, the line transfer problem has 2-D axial symmetry about the binary axis, and the line source function reduces to $S=(1-\varepsilon) \bar{J}+\varepsilon B_{\nu}$.

We are developing an extension of the SEI (Sobolev with Exact Integral) method of Lamers et al. (1987) to solve the binary wind radiative transfer problem. SEI uses escape probability (the Sobolev approximation) to estimate the source function in winds of single stars, followed by an exact formal solution of the radiative transfer equation (the "exact integral"). The formal solution in 
the desired line-of-sight direction will be evaluated using the Feautrier approach (cf. Mihalas 1978). The Sobolev approximation is only valid for monotonic winds with large velocity gradients. Carpenter et al. (1999), in their analysis of the HST/GHRS spectrum of the wind of the $\mathrm{K}$ supergiant $\lambda$ Vel (K4 Ib) compare wind line profiles computed using SEI and an exact comoving frame, CRD calculation. The differences in the emergent profile are small for rapidly accelerating winds $(\beta<1)$, and in particular, SEI is a good approximation for the $\beta=0.9$ wind law that best matches the observations. However, SEI is a poor approximation for slowly-accelerating winds and fails completely in static chromospheres, due to the breakdown of the Sobolev approximation. Recent analyses of the wind of $\zeta$ Aur by Baade et al. (1996) show that the wind out to $5 \mathrm{~K}$-star radii is slowly accelerating $(\beta=3.5)$, a regime for which SEI fails to adequately describe wind line formation.

It is important to realize that the underlying method of SEI is quite powerful in that it relies on general escape probability arguments to compute an approximate source function. Although escape probability methods can be used to compute emergent intensities, first-order methods do this poorly near the boundaries. Using escape probability to estimate the source function followed by an exact formal solution gives much more accurate results for the emergent intensity. The failure of SEI for slow winds and static media is not due to a basic flaw of the method, but rather from the failure of the specific form of the escape probability (the Sobolev approximation) used.

Our approach is to generalize SEI to incorporate escape probability for general spherical media. We will approximate the generalized one-sided escape probability function $\mathcal{P}$ of Hummer \& Rybicki (1982; equation 2.15) by noting that $\mathcal{P}$ is dominated by two asymptotic regimes: the static limit where the velocity gradients are small, and photons escape mainly from the line wings as in the plane-parallel static case:

$$
\mathcal{P}=\mathcal{P}_{K}=\frac{1}{2} K_{2}(\tau) \approx \frac{1}{1+\tau},
$$

and the Sobolev limit where

$$
\mathcal{P}=\mathcal{P}_{S}
$$

and escape is controlled by the velocity gradient. For the plane-parallel, static case, the single-sided escape probability is given by $K_{2} / 2$, where the kernel function,

$$
K_{2}(\tau)=\int_{-\infty}^{\infty} \phi(x) E_{2}[\tau \phi(x)] d x
$$

and $\phi(x)$ is the normalized line profile, $x=\nu / \nu_{D}$ is the dimensionless frequency, and $E_{2}$ is the second exponential integral. The simple $1 /(1+\tau)$ approximation is from Frisch (1984). For the Sobolev regime, we adopt the notation $\mathcal{P}_{S}=\beta$ of Lamers et al. (1987) for the Sobolev escape probability. From Fig. 2 of Hummer \& Rybicki (1982) it is evident that a reasonable approximation to the exact escape probability $\mathcal{P}$ is given by the sum of the static and Sobolev escape probabilities,

$$
\mathcal{P} \approx \mathcal{P}_{K}+\mathcal{P}_{S} \approx \frac{1}{1+\tau}+\beta
$$




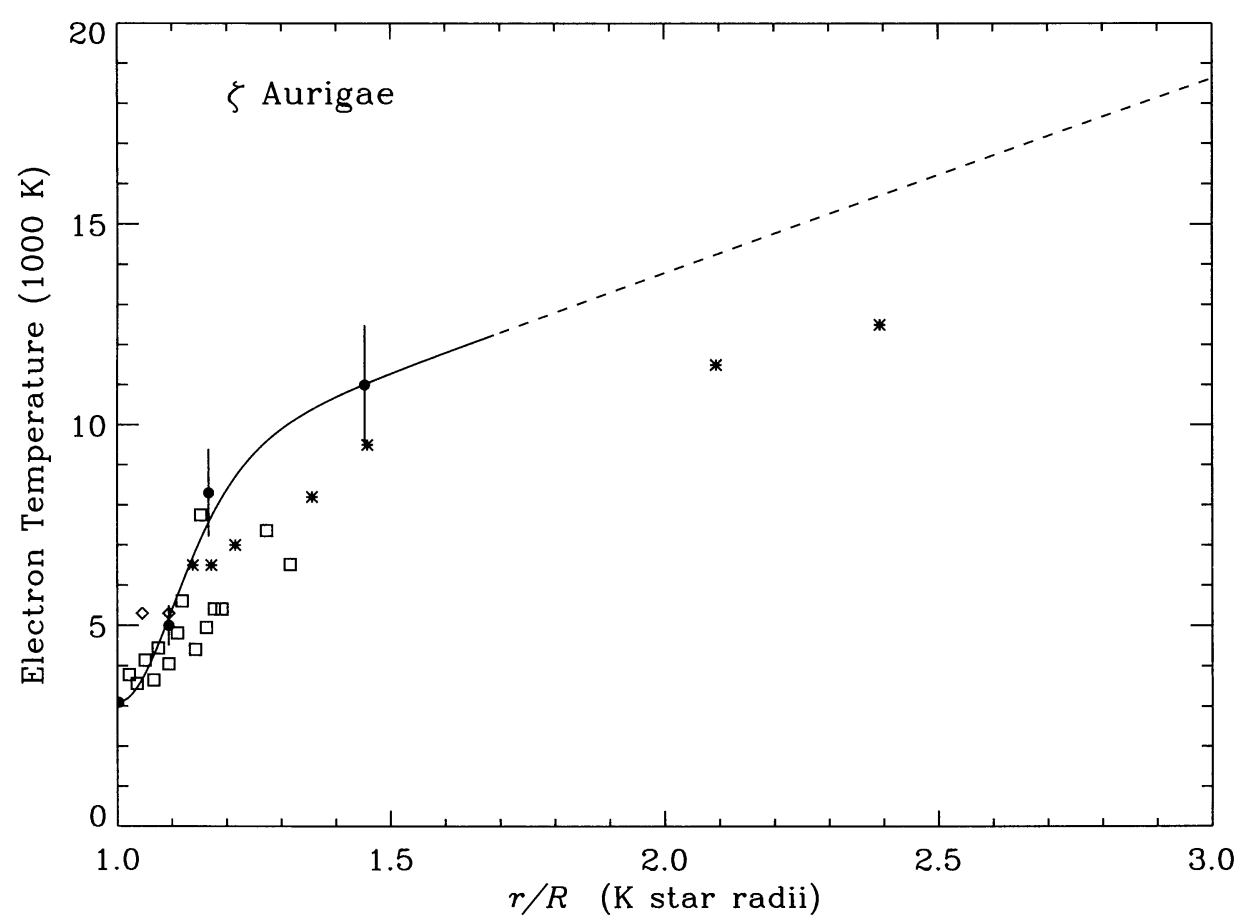

Figure 3. Temperature structure of the chromosphere and wind of the supergiant primary (K4 Ib) of $\zeta$ Aur as inferred from pure absorption analyses for different sightlines. The curve is a smooth fit through our analysis results (filled circles); the dashed portion is a constrained extrapolation into the wind regime where temperatures are $\sim 20000 \mathrm{~K}$ from the observed ionization fractions of $\mathrm{Al} \mathrm{II}$ and $\mathrm{Al}$ III. The asterisks are the results of Eaton (1993). Open diamonds are from the optical analysis of Schröder et al. (1990); the open squares are from Wilson \& Abt (1954).

and this expression forms the basis of our EPEI (Escape Probability with Exact Integral) code for binary stars.

Since we have assumed the wind in the binary systems, is still spherically symmetric with radially-directed velocity vectors, outward photon escape remains spherical. However, the presence of the hot companion introduces a nonspherical boundary condition that breaks the symmetry and renders the problem 2-D axisymmetric instead. The presence of an inner boundary cavity around the hot star introduces another source of escape for trapped photons, and this makes the escape probability problem fundamentally non-spherical and 2-D in nature. Our approach is to use the sum of the outward (radial) and inward (towards the hot star) escape probabilities in approximating the source function. The direct contribution to the source function (the continuum penetration term, $\beta_{c}$, in the notation of Lamers et al. 1987) will be computed using the same optical depths used for the inward escape. The formal solution will be computed using 
the Feautrier method along the (long) characteristics in the desired sightline direction to give emergent intensities.

\section{Some Preliminary Results for $\zeta$ Aur}

To date, we have carried out extensive analyses of the line of sight absorption columns for $\zeta$ Aur, based on HST/GHRS observations. These results were derived assuming pure absorption, based on observed near-eclipse spectra and out-of-eclipse spectra with the eclipse spectrum (the scattering component) subtracted. Because the metastable level populations are close to Boltzmann, an average electron temperature can be derived for each sightline, along with the column density and mean velocity profile. Combining observations from different sightlines yields the variation of electron temperature (Figure 3 ) and tangential column density with radial distance from the supergiant. These analyses suggest the electron temperature of the outer atmosphere of $\zeta$ Aur rises to $\sim 20000 \mathrm{~K}$ at distances of several stellar radii.

However, the situation regarding the wind further from the supergiant is less clear. There are fewer diagnostics available in the wind, and metastable levels (e.g., of Fe III) that are expected to remain close to Boltzmann appear to depopulate. In addition, the "pure absorption" approach breaks down because the scattering component becomes comparable to the absorption component, and it is not obvious how to perform an empirical separation of these components. In this situation, the only recourse is to solve the complete line-formation problem in the wind. Baade et al. (1996) solved the 2-D axisymmetric radiative transfer problem for $\zeta$ Aur for selected lines of Si II and Fe II using an integral operator method. We are now developing the EPEI code outlined in this paper to quickly compute arbitrary line profiles at arbitrary orbital phases of binary systems.

Acknowledgments. Support for this work was provided by NASA through grants GO-03626.01-91A, GO-5398.01-93A, GO-06069.01-94A, GO-07269.01-A, GO-08257.01-A, and GO-09109.01-A to the University of Colorado from the Space Telescope Science Institute, which is operated by the Association of Universities for Research in Astronomy, Incorporated, under NASA contract NAS526555.

\section{Discussion}

COHEN: Are there many binaries of that you can reach with HST? Are the many such spectra?

BENNETT: The binaries that we have observed with HST (GHRS and STIS UV spectrographs) are : $\zeta$ Aur, 32 Cyg, 31 Cyg, HR 2554, VV Cep, KQ Pup. Yes, there is an extensive set of spectra, especially of VV Cep, in the HST archive. 


\section{References}

Ahmad, I. A. 1986, ApJ, 301, 275

Ahmad, I. A., Chapman, R. D., \& Kondo, Y. 1983, A\&A, 126, L5

Ahmad, I. A., \& Stencel, R. E. 1988, ApJ, 329, 797

Baade, R. 1990, A\&A, 233, 486

Baade, R., Kirsch, T., Reimers, D., Toussaint, F., Bennett, P. D., Brown, A., \& Harper, G. M. 1006, ApJ, 466, 979

Busche, J. R., \& Hillier, D. J. 2000, ApJ, 531, 1071

Carpenter, K. G., Robinson, R. D., Harper, G. M., Bennett, P. D., Brown, A., \& Mullan, D. J. 1999, ApJ, 521, 382

Chapman, R. D. 1981 ApJ, 248, 1043

Che, A., Hempe, K., \& Reimers, D. 1983, A\&A, 126, 225

Dullemond, C. P., \& Turolla, R. 2000, A\&A, 360, 1187

Eaton, J. A. 1992, MNRAS, 258, 473

Eaton, J. A. 1993, ApJ, 404, 305

Frisch, H. 1984, in Methods in Radiative Transfer, ed. W. Kalkofen, Cambridge: Cambridge University Press, 65

Griffin, R. E. M., Griffin, R. F., Schröder, K.-P., \& Reimers, D. 1990, A\&A, 234,284

Hempe, K. 1982, A\&A, 115, 133

Hummer, D. G., \& Rybicki, G. B. 1982, ApJ, 254, 767

Kirsch, T. \& Baade, R. 1994, A\&A, 291, 535

Kuin, N. P. M., \& Ahmad, I. A. 1989, ApJ, 344, 856

Lamers, H. J. G. L. M., Cerruti-Sola, M., \& Perinotto, M. 1987, ApJ, 314, 726

Mihalas, D. 1978, Stellar Atmospheres, 2nd ed., San Francisco: Freeman

Schröder, K.-P. 1985, A\&A, 147, 103

Schröder, K.-P. 1986, A\&A, 170, 70

Schröder, K.-P., Griffin, R. E. M., \& Griffin, R. F. 1990, A\&A, 234, 299

Stencel, R. E., \& Chapman, R. D. 1981, ApJ, 251, 597

Wilson, O. C., \& Abt, H. A. 1954, ApJS, 1, 1

Wright, K. O. 1970, Vistas in Astr., 12, 147 